\title{
Die Europäische Ermittlungsanordnung
}

\author{
- Anspruch und wahrscheinliche Wirklichkeit bei einer Umsetzung der \\ geplanten Richtlinie in Deutschland -*
}

\begin{abstract}
Summary
In April 2010 a group of seven Member States of the European Union submitted a draft proposal for a Directive regarding the European Investigation Order in criminal matters. Based on the principle of mutual recognition the directive shall replace all the existing instruments of the traditional system of mutual legal assistance as far as obtaining evidence with a cross-border dimension is concerned. During the ordinary legislative procedure the first reading of the European Parliament is imminent. Taking into account the available information, the future criminal proceeding in cross-border cases in Germany according to the prospective directive can be predicted. A comparison with the currently existing proceeding based on mutual legal assistance allows the assessment that Germany presumably will not reach all of the objectives which are pursued with the Directive regarding the European Investigation Order in criminal matters.
\end{abstract}

\section{Résumé}

Fin avril 2010, un groupe de sept Etats membres de l'Union Européenne a pris des dispositions pour créer une directive concernant la décision d'instruction européenne en matière pénale. Le but de cette directive est, par application du principe de reconnaissance mutuelle des jugements et décisions judiciaires, de remplacer l'actuel système d'entraide judiciaire pour l'obtention de preuves dans des enquêtes à dimension transfrontalière. Il est à noter qu'à l'état actuel, les procédures judiciaires telles que prévues par les cadres législatifs en vigueur dans l'UE, sont déjà largement adaptés. La première lecture du projet pourrait être soumise au Parlement Européen sous peu. Sur la base des informations actuellement disponibles, il est d'ores et déjà possible se faire une idée du déroulement de procédures pénales transfrontalières sur le territoire allemand selon les termes de la future directive. La comparaison avec le système actuel d'entraide judiciaire permet de conclure que certains objectifs partiels de la directive concernant la décision d'instruction européenne en matière pénale probablement ne pourront pas être atteints en Allemagne.

* Der Beitrag enthält Ergebnisse der Masterarbeit des Verfassers, die dieser im Juli 2011 im Fachbereich „Strafrecht, Strafprozessrecht und Kriminalpolitik“" an der Deutschen Hochschule der Polizei, Münster, bei Prof. Dr. Peter Rackow, abschloss. Soweit erforderlich, wurden für diesen Beitrag die relevanten Ergebnisse der Masterarbeit im Lichte der zwischenzeitlichen Entwicklung des Richtlinienentwurfs modifiziert. 


\section{Sachstand des Rechtsetzungsprozesses}

Im Rahmen seiner Tagung am 9./10. Juni 2011 legte der Rat eine partielle allgemeine Ausrichtung des Hauptteils (Art. 1 bis 18 und Art. Y) des Richtlinienentwurfs für eine Europäische Ermittlungsanordnung in Strafsachen (E-RL EEA) fest. ${ }^{1}$ In der Folge befassten sich die Vorbereitungsgremien des Rates intensiv mit den Regelungen der vorgesehenen einzelnen Ermittlungsmaßnahmen des Richtlinienentwurfs. Eine vorläufige Finalisierung erfuhren diese Aktivitäten in der Tagung des Rates am 13./14. Dezember 2011, indem eine Verständigung auf eine allgemeine Ausrichtung des Wortlautes des Richtlinienentwurfs stattfand. ${ }^{2}$ Ausgenommen wurde lediglich die Einigung auf Teile des Artikels 29 sowie einen diesbezüglichen Anhang, die das Verhältnis zu anderen Übereinkünften und Vereinbarungen regeln. Auch die Erwägungsgründe wurden noch nicht vollständig angenommen. ${ }^{3}$

Das Europäische Parlament befasste sich bisher in zwei Sitzungen des Ausschusses für bürgerliche Freiheiten, Justiz und Inneres (LIBE) mit dem Richtlinienentwurf. ${ }^{4}$ Die ursprünglich für den 10. September 2012 anberaumte Erste Lesung (Art. 294 Abs. 3 AEUV), wurde auf einen noch nicht bekannt gewordenen Zeitpunkt verschoben. ${ }^{5}$

Der Rechtsetzungsprozess im Ordentlichen Gesetzgebungsverfahren der EU schreitet also voran und macht eine erneute Befassung mit dem Richtlinienentwurf EEA interessant. Dabei scheint es durchaus lohnenswert, bereits heute einige Schritte voraus zu denken und der Frage nach zu gehen, wie eine zukünftige Richtlinie EEA in deutsches Recht umgesetzt werden könnte, und wie sich hierdurch das bisherige Verfahren justizieller Rechtshilfe hierzulande verändern könnte. Unter Berücksichtigung dessen ließe sich abschätzen, inwieweit einige der Ziele der geplanten Richtlinie in Deutschland tatsächlich zu erreichen sein werden.

1 Ratsdok. 11735/11 vom 17.6.2011.

2 Ratsdok. 18918/11 vom 21.12.2011. Sofern nicht explizit anders ausgewiesen, beziehen sich Fundstellenangaben im Richtlinienentwurf auf diese (bislang letztveröffentlichte) Gesamtfassung.

3 Ratsdok. 18918/11 vom 21.12.2011, S. 1. Den letzten Stand zu den Erwägungsgründen gibt das Ratsdok. 9445/12 vom 29.5.2012 wieder. Nennenswerte Änderungen zur Version der Erwägungsgründe in Ratsdok. 18918/11 vom 21.12.2011 sind jedoch nicht zu verzeichnen.

4 Die Ausschusssitzungen können über die Mediathek des EP in Wort und Bild abgerufen werden. Sitzung vom 12.1.2012: http://www.europarl.europa.eu/ep-live/de/committees/video? event=20120112-1500-COMMITTEE-LIBE. Sitzung vom 26.3.2012: http:/www.europarl.eu ropa.eu/ep-live/de/committees/video? event=20120326-1500-COMMITTEE-LIBE (Zugriff am 4.10.2012).

5 Das ursprüngliche Datum war unter der „Procedure File“ des Europäischen Parlaments: http://www.europarl.europa.eu/oeil/popups/ficheprocedure.do?lang=en\&reference=2010/081 $7 \% 28 C O D \% 29$ noch Ende März (Zugriff am 31.3.2012) ersichtlich. Zwischenzeitlich wird nur noch der Hinweis auf die Erste Lesung angezeigt (Zugriff am 4.10.2012). Nach einer Pressemitteilung des EP vom 14.6.2012 sei die Zusammenarbeit des Parlaments mit dem Rat bei fünf Gesetzesinitiativen, darunter jene zur Richtlinie EEA, ausgesetzt, bis eine Einigung über die Reform des Schengen-Paktes erzielt worden sei. Was unter „Aussetzung“ konkret zu verstehen sei, bleibt unklar. Vgl.: http://www.europarl.europa.eu/news/de/headlines/content/201 20613STO46764/html/EU-Parlament-stoppt-Zusammenarbeit-mit-Rat-nach-Schengen-Entsc heidung (Zugriff am 4.10.2012). 


\section{Begründungszusammenhang und Ziele der zukünftigen Richtlinie EEA}

Die geplante Richtlinie EEA dient der fortgesetzten Verwirklichung des Grundsatzes der gegenseitigen Anerkennung, auf dem gemäß Art. 82 Abs. 1 S. 1 AEUV die justizielle Zusammenarbeit in Strafsachen in der EU beruhen soll. Der im Rahmen der Sondertagung des Europäischen Rates in Tampere im Oktober 1999 als „Eckstein der justiziellen Zusammenarbeit"6 postulierte Grundsatz der gegenseitigen Anerkennung, fand eine Konkretisierung im „Maßnahmenprogramm zur Umsetzung des Grundsatzes der gegenseitigen Anerkennung gerichtlicher Entscheidungen in Strafsachen". ${ }^{7}$ Die dort beschriebenen Maßnahmen fünf und sechs hinsichtlich der Beweiserhebung und des Beweistransfers wurden zunächst durch zwei Rahmenbeschlüsse verwirklicht.

So wurde im Juli 2003 der Rahmenbeschluss Sicherstellung ${ }^{8}$ beschlossen. Dieser Rahmenbeschluss sollte u.a. den Zugriff der Strafverfolgungsbehörden auf Beweismittel, die sich in einem anderen EU-Mitgliedstaat befinden, gewährleisten. Hierfür soll es dem ersuchenden Staat möglich sein, nach dem Grundsatz der gegenseitigen Anerkennung eine Anordnung auf Sicherstellung der Beweismittel im anderen EU-Mitgliedstaat zu erlassen. Die eigentliche Übermittlung der Beweismittel an den ersuchenden Staat richtet sich dann jedoch nach den einschlägigen Regelungen der traditionellen Rechtshilfe. Dies wurde als zu kompliziert und unzureichend erachtet, genauso wie der beschränkte Geltungsbereich nur auf Teile der Beweiserhebung. ${ }^{9}$

Die Kommission schlug deshalb den Rahmenbeschluss über eine Europäische Beweisanordnung ${ }^{10}$ vor, um Beweismittel auf einheitliche, schnelle und effektive Weise erlangen und dem ersuchenden Staat übergeben zu können. Der Erlass einer vorherigen Sicherstellungsanordnung sollte nicht mehr nötig sein. ${ }^{11}$ Ziel des Rahmenbeschlusses über eine Europäische Beweisanordnung sollte es sein, das System der Rechtshilfe in Strafsachen zur Erlangung von Sachen, Schriftstücken oder Daten zwischen den Mitgliedstaaten durch die Anwendung des Grundsatzes der gegenseitigen Anerkennung zu ersetzen. ${ }^{12}$ Der Rahmenbeschluss über eine Europäische Beweisanordnung sollte dabei neben den vorhandenen Rechtshilfeverfahren bestehen. Dies jedoch nur vorläufig, bis eine Ausdehnung auf die noch ausgenommenen Beweisarten erfolgt und ein vollständiges System der gegenseitigen Anerkennung entstanden wäre, das die bisherigen Rechtshilfeverfahren gänzlich ersetzen würde. ${ }^{13}$ Von allen EU-Mitgliedstaaten haben

6 Nr. 33 der Schlussfolgerungen des Vorsitzes, Europäischer Rat (Tampere) vom 15. bis 16. Oktober 1999. Abrufbar unter: http://www.europarl.europa.eu/summits/tam_de.htm (Zugriff am 4.10.2012).

7 ABl. C 12/10 vom 15.1.2001.

8 Rahmenbeschluss 2003/577/JI des Rates vom 22. Juli 2003 über die Sicherstellung von Vermögensgegenständen oder Beweismitteln in der Europäischen Union, ABl. L 196/45 vom 2.8.2003; BGBl. 2008 I, 995.

$9 \operatorname{KOM}(2003) 688$ endgültig vom 14.11.2003, Rn. 23-26.

10 Rahmenbeschluss 2008/978/JI des Rates vom 18. Dezember 2008 über die Beweisanordnung zur Erlangung von Sachen, Schriftstücken und Daten zur Verwendung in Strafsachen, ABl. L 350/72 vom 30.12.2008.

$11 \operatorname{KOM}(2003) 688$ endgültig vom 14.11.2003, Rn. 28.

12 ABl. L 350/72 vom 30.12.2008, Erwägungsgrund 23.

13 ABl. L 350/72 vom 30.12.2008, Erwägungsgrund 25. 
bislang nur Dänemark und Finnland den Rahmenbeschluss über eine Europäische Beweisanordnung umgesetzt. ${ }^{14}$

Ende April 2010 wurde mit der Initiative von sieben Mitgliedstaaten für eine Richtlinie für eine Europäische Ermittlungsanordnung ${ }^{15}$ ein konkreter Vorschlag unterbreitet, der das im vorgenannten Maßnahmenprogramm und im Rahmenbeschluss über eine Europäische Beweisanordnung angekündigte sowie im „Grünbuch Erlangung verwertbarer Beweise in Strafsachen aus einem anderen Mitgliedstaat" 16 anvisierte einheitliche Instrument zur Beweiserhebung in der EU verwirklichen würde. Die Europäische Ermittlungsanordnung soll das durch den Europäischen Rat im „Stockholmer Programm“ geforderte umfassende System für die grenzüberschreitende Beweiserhebung, basierend auf dem Grundsatz der gegenseitigen Anerkennung, werden ${ }^{17}$ und sämtliche diesbezüglichen bestehenden Instrumente (einschließlich des Rahmenbeschlusses über eine Europäische Beweisanordnung) ersetzen. ${ }^{18}$

In der Begründung der Initiative wird ausgeführt, dass der bestehende Rechtsrahmen für die grenzüberschreitende Beweiserhebung fragmentiert und komplex sei. Hauptursache sei das Nebeneinanderbestehen von Rechtsinstrumenten der (traditionellen) Rechtshilfe einerseits und nach dem Grundsatz der gegenseitigen Anerkennung andererseits. ${ }^{19}$ Letztgenannte Rechtsinstrumente seien zahlenmäßig zwar bisher noch gering, jedoch sähen sie sich Kritik ausgesetzt. Unter anderem sei ihre Anwendung kompliziert und ihr Anwendungsbereich zu begrenzt. ${ }^{20}$

Nach Abwägung unterschiedlicher Handlungsvarianten gelangen die Initiatoren zu dem Ergebnis, dass die beste Option zur Lösung der Probleme die Ersetzung aller bestehenden Instrumente durch eine Europäische Ermittlungsanordnung mit umfassendem Anwendungsbereich sei. ${ }^{21}$

Ausweislich des „,Vermerks mit detaillierten Angaben“ zur Initiative für die Richtlinie EEA $^{22}$ wird mit ihr als allgemeines Ziel die Verbesserung der Wahrheitsermittlung in Strafverfahren mit transnationalen Bezügen verfolgt. Dies diene dem Zweck des Aufbaus eines Raumes der Freiheit, der Sicherheit und des Rechts. ${ }^{23}$ Dieses allgemeine Ziel gliedere sich in die folgenden Einzelziele:

1. Beschleunigung des Verfahrens

2. Gewährleistung der Zulässigkeit von Beweismitteln

14 Vgl. Übersicht zum Umsetzungsstand auf der Internet-Seite des EJN: http://www.ejnforum. eu/status_table.php?instrument=1363 (Zugriff am 4.10.2012). Die Umsetzungsfrist endete am 19.1.2011.

15 Initiative des Königreichs Belgien, der Republik Bulgarien, der Republik Estland, des Königreichs Spanien, der Republik Österreich, der Republik Slowenien und des Königreichs Schweden für eine Richtlinie des Europäischen Parlaments und des Rates vom... über die Europäische Ermittlungsanordnung in Strafsachen, ABl. C 165/22 vom 24.6.2010, entspricht Ratsdok. 9145/10 vom 29.4.2010; Interinstitutionelles Dossier: 2010/0817(COD).

$16 \operatorname{KOM}(2009) 624$ endgültig vom 11.11.2009, S. 5.

17 ABl. C 115/12 vom 4.5.2010.

18 Ratsdok. 18918/11 vom 21.12.2011, Erwägungsgrund 6.

19 Ratsdok. 9288/10 ADD 2 vom 3.6.2010, S. 14.

20 Ratsdok. 9288/10 ADD 2 vom 3.6.2010, S. $15 \mathrm{f}$.

21 Ratsdok. 9288/10 ADD 2 vom 3.6.2010, S. $33 \mathrm{ff}$.

22 Ratsdok. 9288/10 ADD 2 vom 3.6.2010.

23 Ratsdok. 9288/10 ADD 2 vom 3.6.2010, S. 21. 
3. Vereinfachung des Verfahrens

4. Beibehaltung eines hohen Niveaus des Schutzes der Grundrechte, insbesondere der Verfahrensrechte

5. Verringerung der finanziellen Kosten

6. Stärkung des gegenseitigen Vertrauens und der Zusammenarbeit zwischen den Mitgliedstaaten

7. Bewahrung der Besonderheiten der nationalen Systeme und der jeweiligen Rechtskultur der Mitgliedstaaten ${ }^{24}$

Es bedarf keiner tiefgehenden Analysen, um zu erkennen, dass zwischen den Teilzielen Wechselwirkungen, bisweilen gar Konkurrenzen bestehen. So kann bspw. die Beschleunigung des Verfahrens zu Lasten eines hohen Schutzniveaus der Grundrechte gehen. Im Zuge der Beratungen um die Richtlinie EEA ein ausgewogenes Verhältnis zwischen den Teilzielen herzustellen, ist damit sicherlich eine echte Herausforderung.

Die nachfolgenden Ausführungen konzentrieren sich auf die Teilziele der Beschleunigung und der Vereinfachung des Verfahrens. Es wird dabei der Versuch unternommen, abzuschätzen, inwieweit diese beiden Ziele nach heutigem Kenntnisstand für Deutschland bei einer Umsetzung der geplanten Richtlinie zu erreichen sein werden.

\section{Erreichbarkeit der Teilziele Beschleunigung und Vereinfachung des Verfahrens in Deutschland}

\section{Bedeutung der Teilziele}

Die Teilziele der Beschleunigung und der Vereinfachung des Verfahrens sind alles andere als selbsterklärend. Ein Blick in die offizielle Begründung hilft nur bedingt weiter. Zur Beschleunigung des Verfahrens wird lediglich auf die Ansprüche einer raschen Aufklärung der Straftat sowie auf den Urteilsspruch innerhalb angemessener Frist verwiesen. Unnötige Verzögerungen müssten vermieden werden. Hinsichtlich der Vereinfachung des Verfahrens wird betont, dass Richter und Staatsanwälte in der EU mittels einfacher Verfahren zusammenarbeiten können sollten. Verfahrensrechtliche Erfordernisse sollten auf das strikt Notwendige begrenzt werden. ${ }^{25}$

Das transnationale Strafverfahren in seiner Gesamtheit ist naturgemäß dadurch gekennzeichnet, dass in mindestens zwei Staaten Verfahrensschritte durchgeführt werden. Dabei kann der Staat einmal Vollstreckungs- und ein andermal Anordnungsstaat sein.

Innerhalb des Verfahrens ist des Weiteren zu unterscheiden zwischen dem Verfahren als solchen und der Bearbeitung im engeren Sinn. Das Verfahren als solches meint die im Einzelnen innerstaatlich zu beteiligenden Stellen und die zwischen ihnen existierenden Geschäftsprozesse. Die Bearbeitung im engeren Sinn beinhaltet hingegen die konkreten Verrichtungen, die die einzelnen beteiligten Stellen zur Sachbearbeitung vollziehen müssen. 
Bezogen auf beide Aspekte lässt die geplante Richtlinie EEA einen mehr oder weniger weiten Handlungsspielraum im Rahmen der Umsetzung auf nationaler Ebene.

\section{2. Änderungsbedarf nationaler Vorschriften}

Richtlinien bedürfen zur Erlangung ihrer Wirksamkeit auf mitgliedstaatlicher Ebene der Umsetzung in nationales Recht, wobei die Wahl der Form und Mittel den innerstaatlichen Stellen überlassen bleibt (Art. 288 S. 4 AEUV). Der Regelungsumfang der Richtlinie EEA wird verhältnismäßig groß sein. Angesichts dessen wäre es denkbar und rechtssystematisch durchaus wünschenswert, die Umsetzung der Richtlinie EEA zum Anlass zu nehmen, ein gänzlich neues deutsches Gesetz zur justiziellen Zusammenarbeit in Strafsachen mit den Mitgliedstaaten der EU zu schaffen - vergleichbar dem österreichischen EU-JZG. ${ }^{26}$ Realistischer ist jedoch, dass der deutsche Gesetzgeber seinem bisherigen Ansatz folgt, und die Regelungen der Richtlinie EEA in das IRG $^{27}$ integrieren wird. ${ }^{28}$ In der Hauptsache dürfte das den Zehnten Teil des IRG (Sonstiger Rechtshilfeverkehr mit Mitgliedstaaten der EU) betreffen. Die Einfügung des Neunten und Zehnten Teils in das IRG im Jahr $2008^{29}$ sollte ausdrücklich „,die zukünftige Umsetzung weiterer Rahmenbeschlüsse erleichtern" “.30

Betroffen sein können weitere einfachgesetzliche Regelungen. In jedem Fall wird jedoch eine Anpassung der ,Richtlinien für den Verkehr mit dem Ausland in strafrechtlichen Angelegenheiten" (RiVASt) ${ }^{31}$ erforderlich werden. Als Verwaltungsvorschrift konkretisieren die RiVASt die Vorschriften des IRG und sind für die betroffenen Gerichte, Staatsanwaltschaften und anderen Behörden verbindlich. ${ }^{32}$

\section{Das Verfahren als solches}

Eine detaillierte Prognose der einzelnen Änderungen ist zum gegenwärtigen Zeitpunkt weder möglich, noch für die Zwecke dieses Beitrages notwendig. Wesentlich aufschlussreicher ist die Antizipation des später rechtlich vorgegebenen Verfahrens zur Umsetzung zukünftig eingehender und ausgehender Europäischer Ermittlungsanord-

26 Bundesgesetz über die justizielle Zusammenarbeit in Strafsachen mit den Mitgliedstaaten der Europäischen Union, österreichisches BGBl. I 2004/36i.d.F. BGBl. I 2004/164, BGB1 I 2007/38, BGB1. I 2007/112; siehe hierzu auch: Kert, in: Vernimmen-Van Tiggelen/Surano/ Weyembergh (Hrsg.): The future of mutual recognition in the European Union, 2009, 15-28.

27 Gesetz über die internationale Rechtshilfe in Strafsachen vom 23.12.1982, BGBl. I, 2071 i.d.F. der Bekanntmachung vom 27.6.1994, BGB1. I, 1537, zuletzt geändert durch Gesetz vom 18.10.2010, BGBl. I, 1408.

28 Vgl. Riegel bei Ronsfeld, ZIS 2012, 636 (640).

29 Die Erweiterung auf den Neunten und Zehnten Teil erfolgte 2008 in Umsetzung des Rahmenbeschlusses Sicherstellung (Fn. 8). Hierzu auch: Jokisch/Jahnke, in: Sieber/Brüner/Satzger/von Heintschel-Heinegg (Hrsg.): Europäisches Strafrecht, 1. Aufl. 2011, § 2, Rn. $6 \mathrm{f}$.

30 BT-Drucks. 16/6563 vom 4.10.2007, S. 10. Anmerkung: Richtlinien konnten in der damaligen sog. Dritten Säule nicht beschlossen werden, weshalb diese hier ungenannt bleiben mussten.

31 BAnz Nr. 196 b vom 24.12.2008.

32 Nr. 1 Abs. 1 RiVASt. Die Bindung der Gerichte findet eine Grenze in der letztlichen gerichtlichen Unabhängigkeit gem. Art. 97 Abs. 1 GG. 
nungen. Nachfolgend werden skizzenhaft der gegenwärtige sowie der zukünftig mögliche innerdeutsche Verfahrensablauf gegenübergestellt.

Dies erfolgt am Beispiel eines eingehenden bzw. ausgehenden Ersuchens bzw. zukünftig entsprechender Anordnungen um Wohnungsdurchsuchung und Beschlagnahme beim Verdächtigen.

\section{a) Deutschland als ersuchter bzw. Vollstreckungsstaat}

aa) Gegenwärtiger Verfahrensablauf

(1) Das ausländische Ersuchen geht bspw. per Post bei der zuständigen Staatsanwaltschaft in Deutschland ein. Welche die zuständige Staatsanwaltschaft ist, wurde vorher durch die ausländische ersuchende Staatsanwaltschaft mit Hilfe des ATLAS auf der EJN-Internetseite ermittelt. Dem Ersuchen beigefügt sind die Anordnungen des ersuchenden Staates (bspw. richterlicher Durchsuchungsbeschluss).

(2) Die Staatsanwaltschaft ${ }^{33}$ prüft, ob das Ersuchen ordnungsgemäß erledigt worden ist (Nr. 7 lit. b RiVASt) und, sofern alle Voraussetzungen für die Leistung von Rechtshilfe vorliegen, bewilligt das Ersuchen um Wohnungsdurchsuchung und Beschlagnahme (§ 59 IRG i.V.m. einschlägigem Übereinkommen, Nr. 7 lit. a RiVASt). Die Staatsanwaltschaft hat die notwendigen richterlichen Anordnungen zu erwirken (Nr.75 1. Hs. RiVASt). Sie leitet einen entsprechenden Antrag unter Beifügung des Ersuchens an das zuständige Amtsgericht.

(3) Das Amtsgericht ( $\$ 67$ Abs. 3 IRG) prüft auf Grundlage des Ersuchens, ob die Voraussetzungen für eine Wohnungsdurchsuchung und Beschlagnahme nach deutschem Recht vorliegen. Grundlage der Prüfung sind dabei die in dem Ersuchen (einschl. der übermittelten Anordnungen des ersuchenden Staates) mitgeteilten Tatsachen. Das Gericht ordnet bei positiver Prüfung die Wohnungsdurchsuchung ( $\$ 67$ Abs. 1 IRG i.V.m. $\S \S 102$ ff. StPO) und Beschlagnahme (§ 67 Abs. 1 IRG i.V.m. $\S \S 94$ ff. StPO) an. Sodann leitet das Gericht die Anordnung auf dem üblichen Geschäftsweg an die Staatsanwaltschaft.

(4) Die Staatsanwaltschaft hat für die Durchführung der erbetenen Maßnahmen zu sorgen (Nr. 75 2. Hs. RiVASt) und leitet die richterliche Anordnung nebst Ersuchen und ggf. weiteren Unterlagen auf dem üblichen Geschäftsweg an die zuständige Polizeidienststelle.

(5) Die Polizei führt die Wohnungsdurchsuchung unter Berücksichtigung der deutschen Form- und Verfahrensvorschriften (§ 77 Abs. 1 IRG i.V.m. u.a. $\S \S 104,106,107$ StPO) sowie ggf. den durch den ersuchenden Staat mitgeteilten Formvorschriften durch (Nr. 22 Abs. 1 RiVASt). Die sichergestellten bzw. beschlagnahmten Gegenstände werden nebst den entsprechenden Unterlagen der Staatsanwaltschaft übergeben (Nr. 23 Abs. 1 RiVASt).

33 Am Beispiel Rheinland-Pfalz: § 74 Abs. 2 IRG i.V.m. Zuständigkeitsvereinbarung (BAnz S. 11494), Nr.1 i.V.m. Verwaltungsvorschrift JM 9350-4-61 vom 30. Juli 2004, Nr. 2.1 i.V.m. Verwaltungsvorschrift JM 9350-4-62 vom 9. August 2004, Nr. 1.2. 
(6) Die Staatsanwaltschaft ( $\$ 66$ Abs. 4 IRG) veranlasst sodann die Bewilligung der Herausgabe der Gegenstände und stellt hierfür einen entsprechenden Antrag bei der örtlich zuständigen Generalstaatsanwaltschaft.

(7) Die Generalstaatsanwaltschaft ${ }^{34}$ prüft und bewilligt die Herausgabe der Gegenstände (§66 IRG) und teilt dies der Staatsanwaltschaft mit.

(8) Die Staatsanwaltschaft übermittelt die beschlagnahmten Gegenstände nebst entsprechenden Unterlagen unmittelbar an die ersuchende Staatsanwaltschaft (§66 Abs. 4 Satz 1 IRG; Nr. 76 RiVASt; Nr. 23 Abs. 2 RiVASt).

\section{bb) Möglicher zukünftiger Verfahrensablauf}

Schritt (1) ändert sich insoweit, dass bei der Staatsanwaltschaft als zuständiger Vollstreckungsbehörde kein Ersuchen, sondern eine Anordnung mittels Formblatt eingeht, die explizit auf Wohnungsdurchsuchung und Beschlagnahme bestimmter Gegenstände beim Beschuldigten lautet. Die nach dem Recht des Anordnungsstaates erforderlichen Beschlüsse etc. sind nicht beigefügt.

Inwieweit sich der weitere Verfahrensablauf ändern wird, ist im Wesentlichen abhängig von der Auslegung der Bestimmungen des Art. 8 Abs. 1 E-RL EEA. Entscheidend ist das Verständnis des ersten Teils des Art. 8 Abs. 1 E-RL EEA, wonach die Vollstreckungsbehörde die EEA ,ohne jede weitere Formalität" anerkennt und ,für deren Vollstreckung in derselben Weise und unter denselben Modalitäten“ sorgt, ,als wäre die betreffende Ermittlungsmaßnahme von einer Behörde des Vollstreckungsstaats angeordnet worden".

Nach dem Grundsatz der Gesetzmäßigkeit (Art. 20 Abs. 3 GG) bedürfen staatliche Grundrechtseingriffe, und hierauf wäre die EEA gerichtet, unbedingt einer gesetzlichen Ermächtigungsgrundlage. Die Formulierung „,ohne jede Formalität“ kann deshalb nicht so aufgefasst werden, dass es keiner Leistungs- und Vornahmeermächtigung in Deutschland bedarf und eine diesbezügliche Prüfung unterlassen werden müsste. Es sind dabei mehrere Varianten denkbar:

\section{Variante 1 - enge Auslegung des Art. 8 Abs. 1 E-RL EEA}

In enger Auslegung des Art. 8 Abs. 1 E-RL EEA würde beispielsweise in Teil Zehn IRG eine Norm eingefügt, die gleichzeitig Leistungs- und Vornahmeermächtigung für die deutschen Behörden wäre. Diese Norm würde sinngemäß lauten, dass einer Anordnung auf Grundlage der Richtlinie EEA zu entsprechen ist und die angeordnete Maßnahme zu ergreifen ist; für die Durchführung wären die nationalen Formvorschriften und Verfahren analog anzuwenden sowie die diesbezüglichen Anforderungen gemäß Anordnung zu beachten. ${ }^{35}$

34 Am Beispiel Rheinland-Pfalz: § 74 Abs. 2 IRG i.V.m. Zuständigkeitsvereinbarung (BAnz S. 11494), Nr. 1 i.V.m. Verwaltungsvorschrift JM 9350-4-61 vom 30. Juli 2004, Nr. 2.1 i.V.m. Verwaltungsvorschrift JM 9350-4-62 vom 9. August 2004, Nr. 1.1.

35 Auf Ausführungen zu Versagungs- und Aufschubgründen, sowie Regelungen zu Alternativmaßnahmen und spezifischen Ermittlungsmaßnahmen kann zur Darstellung des grundsätzlichen Ablaufes hier verzichtet werden. 
Im Beispielfall würde dies bedeuten, die Staatsanwaltschaft behandelt die ausländische Anordnung der Wohnungsdurchsuchung und Beschlagnahme beim Verdächtigen fiktiv als jene eines deutschen Gerichtes. Mit dieser Gleichsetzung entfielen Teile des bisherigen Schrittes (2) sowie Schritt (3), also die deutsche richterliche Anordnung als Erlass einer deutschen Vornahmeermächtigung. Die Staatsanwaltschaft würde die Polizei unmittelbar mit der Durchführung der Wohnungsdurchsuchung und der Beschlagnahme der gesuchten Gegenstände beauftragen (Schritt 4). Für die Durchführung der Wohnungsdurchsuchung und der Beschlagnahme durch die Polizei (Schritt 5) ändert sich grundsätzlich nichts, außer dass die Polizei auf einer anderen Ermächtigungsgrundlage als bisher hin tätig wird (neue Vornahmeermächtigung aus Teil Zehn IRG i.V.m. ausländischer EEA). Die Bewilligung der Herausgabe der aufgefundenen Beweismittel (Schritt 6 und 7) würde, konsequent gedacht, entfallen. Leistungs- und Vornahmeermächtigung zur Herausgabe wäre insofern ebenfalls die oben skizzierte neue Norm im IRG. Eine explizite Prüfung könnte gleichwohl entfallen. An der Übermittlung der beschlagnahmten Gegenstände (Schritt 8) würde sich grundsätzlich nichts ändern.

\section{Variante 2 - weite Auslegung des Art. 8 Abs. 1 E-RL EEA}

In einer weiten Auslegung ${ }^{36}$ würde der betreffende Abschnitt des Art. 8 Abs. 1 E-RL EEA (,,in derselben Weise und unter denselben Modalitäten “) ${ }^{37}$ so verstanden, dass hierunter die umfassende Beachtung der nationalen Regelungen gefasst werden kann. Im Falle Deutschlands also, bezogen auf das Beispiel, die Beachtung sämtlicher einschlägiger rechtlicher Regelungen und höchstrichterlicher Entscheidungen zu Wohnungsdurchsuchung und Beschlagnahme. Art. 8 Abs. 1 E-RL EEA wäre demnach die EU-rechtliche Legitimation zur Beibehaltung bestehender innerstaatlicher Regelungen. Es bedürfte insoweit einer Änderung des IRG, dass bspw. in Teil Zehn eine Leistungs- und Vornahmeermächtigung in Bezug auf Anordnungen gemäß Richtlinie EEA aufgenommen wird. Diese würde beinhalten, dass für die Vornahme die entsprechenden Ermächtigungsgrundlagen (hauptsächlich der StPO) zu Grunde zu legen sind.

Damit bliebe es grundsätzlich beim bisherigen Ablauf(Schritte 2 bis 7), insbesondere hinsichtlich des Erfordernisses eines Durchsuchungs- und Beschlagnahmebeschlusses eines deutschen Gerichts. Bezüglich der Übermittlung der beschlagnahmten Gegenstände (Schritt 8) würde sich, wie in Variante 1 auch, grundsätzlich nichts ändern.

\section{Variante 3 - Mischformen}

Ausgehend von Variante 1 und Variante 2 sind auch Mischformen unterschiedlicher Ausprägung möglich. Im Verhältnis zu den heutigen Verfahren wäre es beispielsweise

36 Zur Erstellung der Masterarbeit wurden Interviews u.a. mit Mitarbeitern von Bundesministerien geführt, die in die Verhandlungen zum Richtlinienentwurf EEA involviert waren. Die o.g. Auslegung wurde in einem dieser Interviews vertreten.

37 Englische Textfassung: ,in the same way and under the same modalities“; französische Textfassung: „de la même manière et suivant les mêmes modalités“. 
denkbar, Umfang und Tiefe der richterlichen Prüfung einzugrenzen oder die gesonderte Bewilligung der Herausgabe der Gegenstände (Schritt 6 und 7) entfallen zu lassen.

\section{cc) Bewertung}

Wie aus der vorangegangen Darstellung deutlich wird, wäre für Deutschland nur in Variante 1 oder abgestuft in Variante 3 eine nennenswerte Veränderung der Verfahrensabläufe zu erwarten. Gleichfalls deuten die Umsetzung der bisherigen Instrumente nach dem Grundsatz der gegenseitigen Anerkennung in Deutschland - allen voran die Umsetzung des $\mathrm{RB} \mathrm{EuHb}^{38}$ - darauf hin, dass die Umsetzung der geplanten Richtlinie EEA Variante 2 folgen wird. Es bliebe damit im Grunde bei der Zweistufigkeit des Verfahrens (Bewilligung und Vornahme) und den dementsprechenden Verantwortlichkeiten und Abläufen. Im Ergebnis wäre damit eine Beschleunigung und Vereinfachung des Verfahrens bei eingehenden Anordnungen nicht zu erreichen.

\section{b) Deutschland als ersuchender bzw. Anordnungsstaat}

\section{aa) Gegenwärtiger Verfahrensablauf}

(1) Im Rahmen der Ermittlungen stellt sich heraus, dass der Beschuldigte über eine Wohnung in einem anderen EU-Mitgliedstaat verfügt. Es besteht die begründete Annahme, dass dort beweiserhebliche Gegenstände aufzufinden sind. Die Polizei regt daraufhin, analog einem rein innerstaatlichen Fall, bei der ermittlungsführenden Staatsanwaltschaft die Stellung eines Ersuchens um eine Wohnungsdurchsuchung und Beschlagnahme an.

(2) Die Staatsanwaltschaft prüft das Vorliegen der Voraussetzungen für eine Wohnungsdurchsuchung und Beschlagnahme nach deutschem Recht ( $\$ 102$ ff., 94 ff. StPO) sowie das Vorliegen der rechtshilferechtlichen Voraussetzungen (Nr. 25 RiVASt i.V.m. einschlägigen Übereinkünften). Nach positiver Prüfung beantragt sie eine entsprechende richterliche Anordnung beim zuständigen Amtsgericht.

(3) Das Amtsgericht ordnet nach Bejahung der rechtlichen Voraussetzungen die Wohnungsdurchsuchung und Beschlagnahme an ( $\S \S 102 \mathrm{ff} ., 94 \mathrm{ff}$. StPO). Es leitet die Anordnung(en) an die Staatsanwaltschaft weiter.

(4) Die Staatsanwaltschaft prüft abschließend das Vorliegen aller rechtshilferechtlichen Voraussetzungen (Nr. 25 RiVASt i.V.m. einschlägigen Übereinkünften) und be-

38 Rahmenbeschluss 2002/584/JI des Rates vom 13. Juni 2002 über den Europäischen Haftbefehl und die Übergabeverfahren zwischen den Mitgliedstaaten, ABl. L 190/1 vom 18.7.2002; BGBl. 2006 I, 1721. Bei Umsetzung des RB EuHb hielt Deutschland rahmenbeschlusswidrig an der Zweistufigkeit des Auslieferungsverfahrens fest; von Heintschel-Heinegg, in: Sieber/ Brüner/Satzger/von Heintschel-Heinegg (Hrsg.), siehe Fn. 29, § 37, Rn. 34. Siehe auch: Hackner, in: Schomburg/Lagodny/Gleß/Hackner (Hrsg.): Internationale Rechtshilfe in Strafsachen, 5. Aufl. 2012, HT III A 1, Rn. 1 ff. 
willigt das Ersuchen. ${ }^{39}$ Sie fertigt das Ersuchen unter Berücksichtigung der einschlägigen Regelungen ordnungsgemäß aus (Nr. 25 bis 31, 114 RiVASt).

(5) Das Ersuchen nebst Unterlagen wird der zuständigen Justizbehörde im betreffenden EU-Mitgliedstaat übermittelt (Nr. 30 Abs. 2 und 3 RiVASt). Welche Behörde dort die zuständige ist, hat die Staatsanwaltschaft über den ATLAS auf der EJN-Internetseite oder u.U. über die EJN-Kontaktstelle ihres Bundeslandes ermittelt. Die Übermittlung erfolgt unmittelbar bspw. per Post oder Fax.

bb) Möglicher zukünftiger Verfahrensablauf

Für den Erlass einer ausgehenden EEA ist nicht ersichtlich, dass sich der bisherige Verfahrensablauf (Schritte 1 bis 5) ändern würde. Es bliebe insbesondere beim Erfordernis, in Deutschland einen richterlichen Beschluss für die Wohnungsdurchsuchung und Beschlagnahme zu erwirken.

\section{cc) Bewertung}

Für die Fälle, in denen Deutschland Anordnungsstaat sein wird, sind für das innerstaatliche Verfahren keine Veränderungsnotwendigkeiten erkennbar, weshalb auch dort weder eine Beschleunigung noch eine Vereinfachung des Verfahrens als solchem erwartbar ist.

\section{Die Bearbeitung im engeren Sinn}

\section{a) Begrenzung der Versagungsgründe}

Wesentlicher Anspruch der Initiative zur Schaffung einer Richtlinie EEA ist, die Effizienz der justiziellen Zusammenarbeit in Strafsachen sicherzustellen. Dies vor allem, indem die Möglichkeiten einer Versagung der Anerkennung oder Vollstreckung der EEA beschränkt werden (Erwägungsgrund 12). Bei Ergreifung der Initiative war die Rede von einer „strenge(n) Begrenzung der Versagungsgründe“, 40 die auf vier Fälle beschränkt sein sollte. Diesem Anspruch kann die derzeitige Entwurfsfassung in Folge zahlreicher Änderungen seit Ergreifung der Initiative im April 2010 nicht mehr gerecht werden. ${ }^{41}$

Die Gründe für die Versagung der Anerkennung oder der Vollstreckung einer EEA sind abschließend in Art. 10 E-RL EEA genannt. Es handelt sich dabei um eines der am intensivsten diskutierten Themen in den Vorbereitungsgremien des Rates. ${ }^{42}$ Der im maßgeblichen Zeitraum amtierende ungarische Ratsvorsitz räumte ein, dass Artikel 10 möglicherweise auf den ersten Blick komplex wirke. Dies sei jedoch dem herausfor-

39 Am Beispiel Rheinland-Pfalz: § 74 Abs. 2 IRG i.V.m. Zuständigkeitsvereinbarung (BAnz S. 11494), Nr. 1 i.V.m. Verwaltungsvorschrift JM 9350-4-61 vom 30. Juli 2004, Nr. 2.1 i.V.m. Verwaltungsvorschrift JM 9350-4-62 vom 9. August 2004, Nr. 2.2.

40 Ratsdok. 9288/10 ADD 1 vom 3.6.2010, zu Art. 10.

41 Vgl. Rackow, in: Ambos (Hrsg.): Europäisches Strafrecht post-Lissabon, 2011, 117 (131 f.).

42 Vgl. Ratsdok. 8474/11 vom 19.4.2011, S. 2 ff. 
dernden Ziel geschuldet, sämtliche bisher existierenden einschlägigen Regelungen in einem Instrument zusammenfassen zu wollen. Der die Versagungsgründe beinhaltende Art. 10 E-RL EEA müsse in unmittelbarem Zusammenhang mit Art. 9 E-RL EEA gesehen werden, der den Rückgriff auf eine alternative Maßnahmen, als die in der EEA angeordnete, regelt. ${ }^{43}$

Eine zukünftig ausgestellte Ermittlungsanordnung wird die ersuchte(n) Ermittlungsmaßnahme(n) explizit benennen, die durch die Vollstreckungsbehörde ergriffen werden soll(en).${ }^{44}$ Der mit der jeweiligen Ermittlungsanordnung verfolgte Zweck soll im rechtlichen Rahmen des Vollstreckungsstaates, ggf. unter Beachtung von Formvorschriften und Verfahren des Anordnungsstaates, so weit wie möglich erreicht werden. Bevor also eine Versagung erteilt wird, sind alle anderen Möglichkeiten der Umsetzung, expressis verbis die Durchführung von Alternativmaßnahmen (Art. 9 E-RL EEA), zu prüfen.

Eine nähere Betrachtung der maßgeblichen Art. 9 und 10 E-RL EEA lässt erahnen, dass die konkreten Bearbeitungsschritte zur Prüfung insbesondere eingehender Anordnungen sich alles andere als trivial darstellen werden. Das Ausmaß einer möglichen Vereinfachung und Verringerung von Arbeitsaufwänden in der Bearbeitung im engeren Sinn in Folge der Einschränkung tradierter Rechtshilfehindernisse wird deshalb nur relativ gering ausfallen. Das Verfahren nach dem Grundsatz der gegenseitigen Anerkennung ist in jedem Fall nicht mit einer Quasi-Automatisierung gleichzusetzen. Das ursprüngliche Ziel einer möglichst weitgehenden Reduzierung von Versagungsgründen und damit einfacheren und zügigeren Bearbeitung wird also nur bedingt erreicht werden können.

\section{b) Nutzung von Formblättern}

Der Richtlinienentwurf sieht die Übermittlung der Europäischen Ermittlungsanordnung mittels Formblatt vor (Art. 5 Abs. 1 E-RL EEA). ${ }^{45}$ Das Formblatt soll das einzige zu übermittelnde Dokument sein. ${ }^{46}$ Dieses Vorgehen ist auf der Linie der bisherigen Rechtsakte nach dem Grundsatz der gegenseitigen Anerkennung. In Deutschland fand beispielsweise das Formblatt des $\mathrm{RB} \mathrm{EuHb}{ }^{47}$ als Vordruck Nr. 40 Eingang in die RiVASt. ${ }^{48}$ Der Vordruck wird in einer Untersuchung als selbsterklärend und einfach auszufüllen beschrieben. Darüber hinaus kommt in einigen Anordnungsbehörden eine Software zum Einsatz, die neben einer Ausfüllhilfe eine automatische Übersetzung der Standardtexte erlaubt. ${ }^{49}$

Die Verwendung von Formblättern vermag Arbeitsabläufe zu verbessern. Bei elektronischer Umsetzung werden Datenbrüche vermieden und eine zügige Datenübermittlung sowie die Nutzung weiterer Funktionalitäten ermöglicht. Im Vergleich zu den bis-

43 Ratsdok. 10749/2/11 REV 2 vom 8.6.2011, S. 3.

44 Vgl. Ratsdok. 9445/12 vom 29.5.2012, Anhang II (Formblatt EEA), Abschnitt C.

45 Den letzten Stand zu den Formblättern gibt das Ratsdok. 9445/12 vom 29.5.2012, Anhang II, wieder.

46 Vgl. Ratsdok. 9288/10 ADD 1 vom 3.6.2010, zu Art. 5.

47 Art. 8 Abs. 1 RB EuHb, Anhang Europäischer Haftbefehl.

48 Nr. 8 Abs. 3 RiVASt erklärt die Verwendung von Vordrucken als zulässig.

49 Ratsdok. 7058/1/09 REV 1, S. 9 f. 
herigen, teilweise umfängliche protokollarische Bekundungen beinhaltenden Rechtshilfeersuchen, erleichtern die Formblätter das schnelle Erfassen des Sachverhalts und des Anliegens. ${ }^{50}$

Jedoch limitieren die Formblätter den Umfang der möglichen Angaben, was insbesondere bei komplexeren Sachverhalten zu nicht unerheblichen Informationseinbußen führen kann. Dem Formblatt müssen - anders als in der traditionellen Rechtshilfe - nicht die (ausführlicheren) Beschlüsse oder Anordnungen beigefügt werden. Damit ist der Informationsgehalt der übermittelten Unterlagen im Verhältnis zum bestehenden System nochmals vermindert. Dies erschwert es den Behörden des Vollstreckungsstaates, die notwendigen Prüfungen vorzunehmen. Gerade bei grundrechtsintensiven Maßnahmen kann sich die Prüfung der im Ausland entscheidungsrelevanten Tatsachen, der Beweiserheblichkeit und der Verhältnismäßigkeit damit nur noch auf eine sehr schmale Basis gründen. ${ }^{51}$

Im Zweifel müssten im Zuge der Prüfung Nachfragen bei der Anordnungsbehörde gestellt werden, um im Einzelfall Informationslücken auf Grund des inhaltsreduzierten Formblattes zu schließen.

Den Vorteilen der Nutzung von Formblättern stehen also eine Reduktion des Informationsumfangs und damit eine Begrenzung von Prüfungsmöglichkeiten gegenüber, was sich gleichwohl konsistent in das Bild des Grundsatzes der gegenseitigen Anerkennung einfügt, der sich auf gegenseitiges Vertrauen stützen soll.

\section{c) Fristen}

Die Beschleunigung des Verfahrens soll durch Einführung spezifischer Fristen für alle Maßnahmenarten gewährleistet werden. ${ }^{52}$ Gemäß Art. 11 Abs. 1 E-RL EEA sind die EU-Mitgliedstaaten grundsätzlich angehalten, möglichst zügig aktiv zu werden. Die Entscheidung über die Anerkennung oder Vollstreckung und die Durchführung der Ermittlungsmaßnahme soll genauso rasch und vorrangig erfolgen, wie in einem vergleichbaren innerstaatlichen Fall. Die hiermit festgelegte Gleichstellung EU-Ausland zum Inland stellt eine Neuerung dar. Die Befolgung dieses Grundsatzes soll nach Ansicht der Initiatoren die meisten der gegenwärtigen Verzögerungen in Rechtshilfeverfahren zukünftig vermeiden können. ${ }^{53}$

Über die Anerkennung und Vollstreckung einer EEA soll so bald wie möglich, jedoch spätestens 30 Tage nach Eingang der Anordnung bei der zuständigen Vollstreckungsbehörde entschieden werden. Im Einzelfall soll eine Fristverlängerung um höchstens 30 Tage möglich sein. Die Vollstreckungsbehörde soll die Ermittlungsmaßnahme(n) un-

50 Das EJN empfiehlt im Ergebnis einer Abfrage in allen EU-Mitgliedstaaten die generelle Verwendung von Formblättern, Ratsdok. 5684/09 vom 26.1.2009, S. 10.

51 Wahl, in: Vernimmen-Van Tiggelen/Surano/Weyembergh (Hrsg.), siehe Fn. 26, 115 (121); Roger, GA 2010, 27 (37); Vgl. Esser, in Schünemann/Jäger u.a. (Hrsg.): Festschrift für Claus Roxin zum 80. Geburtstag, 2011, 1497 (1501f.), mit einer detaillierten Darstellung der Schwierigkeiten, denen sich ein deutscher Richter bei Anordnung einer Wohnungsdurchsuchung zur Vollstreckung einer Europäischen Beweisanordnung gegenüber sähe.

52 Ratsdok. 9288/10 ADD 2 vom 3.6.2010, S. 37.

53 Ratsdok. 9288/10 ADD 1 vom 3.6.2010, zu Art. 11. 
verzüglich durchführen. Als Maximalfrist bestimmt der Richtlinienentwurf 90 Tage nach der Entscheidung über die Anerkennung der Anordnung. Besondere (u.U. kürzere) Verfahrensfristen sollen durch die Vollstreckungsbehörde ,möglichst weitgehend berücksichtigt" werden (Art. 11 Abs. 2 E-RL EEA). Die erlangten Beweismittel sollen grundsätzlich ,ohne unnötige Verzögerung“ an den Anordnungsstaat übermittelt werden (Art. 12 Abs. 1 E-RL EEA).

Der Einführung verpflichtender Fristen liegt die Annahme zu Grunde, dass Rechtshilfeersuchen (zumindest teilweise) zügiger bearbeitet werden könnten, als es gegenwärtig getan wird. Fraglich ist erstens, inwieweit diese Annahme stimmt, und zweitens, inwieweit formale Fristsetzungen geeignet sind, diese Situation zu verbessern. Es ist sicherlich nicht auszuschließen, dass in bestimmten Fällen in einzelnen EU-Mitgliedstaaten vermeidbare Verzögerungen entstehen oder in Kauf genommen werden, nur deshalb, weil es sich um ein Verfahren des Auslandes handelt. Die Ursachen hierfür können mannigfaltig sein. Sie dürften in aller Regel jedoch den gleichen Mustern folgen, wie in der Bearbeitung rein innerstaatlicher Fälle. Maßgeblich können innerstaatliche rechtliche Vorgaben (Legalitätsprinzip vs. Opportunitätsprinzip) genauso sein, wie für die Behörden verbindliche Prioritätenentscheidungen der Kriminalpolitik. Hier kann es Abweichungen zwischen den EU-Mitgliedstaaten geben, die sich auch in der Bearbeitungsdauer niederschlagen. Es wäre jedenfalls nicht einzusehen, dass einem ausländischen Verfahren in einem vergleichbaren Fall der Vorzug vor einem inländischen Verfahren gegeben werden sollte. Der Grundsatz eines möglichst schnellen Verfahrens gilt generell. ${ }^{54}$ Die Ursachen für eine langsamere Bearbeitung liegen regelmäßig nicht in rechtlichen Vorgaben oder im Ermessen der zuständigen Behörden, sondern schlicht in den tatsächlichen Gegebenheiten. Dabei kommt den zur Verfügung stehenden Ressourcen (personell, materiell und finanziell) die größte Bedeutung zu. ${ }^{55}$ An dieser Ursache können Fristen genau so wenig verändern, wie am unter Umständen vorhandenen Unwillen einzelner Verantwortlicher, (frühzeitig) aktiv zu werden.

Der Richtlinienentwurf EEA enthält darüber hinaus keinen Hinweis darauf, ob und wenn ja, welche Konsequenzen bei Nichteinhaltung der Fristen folgen sollen. Es ist indes auch nicht absehbar, dass ein wirksamer Durchsetzungsmechanismus geschaffen wird. Ob bei wiederholten oder dauerhaften Fristverstößen die Einleitung eines Vertragsverletzungsverfahren gem. Art. 258 AEUV in Erwägung gezogen wird, bleibt abzuwarten. Insofern ist die Einführung von Fristen zwar zu begrüßen, ihre Wirkung dürfte jedoch auf den eines Appells beschränkt bleiben.

\section{d) Bestätigungs-, Konsultations- und Unterrichtungspflichten}

Der Richtlinienentwurf sieht die Einführung verschiedener Bestätigungs-, Konsultations- und Unterrichtungspflichten vor. Beispielhaft können genannt werden: die Verpflichtung zur Unterrichtung der Anordnungsbehörde, sollte die Ermittlungsanordnung an eine unzuständige Stelle adressiert worden sein (Art. 6 Abs. 5 E-RL EEA); die gegenseitige Konsultation der Vollstreckungs- und Anordnungsbehörden zur effizienten

54 Art. 6 Abs. 1 S. 1 EMRK.

55 So auch Wahl (Fn. 51), 115 (136). 
Anwendung der einschlägigen Regelungen (Art. 8 Abs. 4 E-RL EEA); die Unterrichtung der Anordnungsbehörde sofern die Vollstreckungsbehörde auf eine andere als in der Ermittlungsanordnung genannte Ermittlungsmaßnahme zurück greifen möchte oder muss (Art. 9 Abs. 2 E-RL EEA) bzw. die Mitteilung an die Anordnungsbehörde, wenn die erbetene Unterstützung aus bestimmten Gründen nicht geleistet werden kann (Art. 9 Abs. 3 E-RL EEA); die Konsultation der Anordnungsbehörde in den Fällen, in denen die Anerkennung oder Vollstreckung der Ermittlungsanordnung teilweise oder ganz versagt werden soll (Art. 10 Abs. 2 E-RL EEA) oder schließlich die Unterrichtung der Anordnungsbehörde über die nicht fristgerechte Bearbeitung der Ermittlungsanordnung sowie die Mitteilung der voraussichtlichen Entscheidungsdauer (Art. 11 Abs. 5 E-RL EEA).

Einzelne solcher Verpflichtungen existieren bereits gegenwärtig. So enthält beispielsweise Nr. 17 RiVASt die Regelung, bei einer Fehladressierung die ersuchende Stelle entsprechend zu benachrichtigen. Das Ausmaß an Bestätigungs-, Konsultations- und Unterrichtungspflichten im Richtlinienentwurf ist gleichwohl größer als im bisherigen rechtshilferechtlichen Verfahren.

Ohne es an dieser Stelle konkret beziffern zu können, ist absehbar, dass hierdurch die verwaltungsmäßigen Aufwände steigen werden.

\section{Zusammenfassende Bewertung}

Die geplante Richtlinie EEA bietet die Möglichkeit, die innerstaatlichen Abläufe des tradierten Rechtshilfeverfahrens auf den Grundsatz der gegenseitigen Anerkennung hin anzupassen und - durch die weitest mögliche Ausschöpfung aller mit der Richtlinie EEA geschaffenen Potentiale für eine Beschleunigung und Vereinfachung des transnationalen Strafverfahrens - zu optimieren.

Entscheidend hierfür wäre die konsequente Verwirklichung des Grundsatzes der gegenseitigen Anerkennung, indem auf die Umwandlung der justiziellen Entscheidung (mit mindestens gerichtlicher Validierung gem. Art. 1 Abs. 1 S. 1 E-RL EEA) eines anderen EU-Mitgliedstaates in eine innerstaatliche Entscheidung vor ihrer Vollstreckung verzichtet wird. Hierdurch könnte bei zukünftigen eingehenden Anordnungen durch die Verringerung notwendiger Verfahrensschritte ein Effizienzgewinn erzielt werden; bei ausgehenden Anordnungen wird dies im innerstaatlichen Verfahren nicht zu erwarten sein. Bei keinem der bisherigen Rechtsakte nach dem Grundsatz der gegenseitigen Anerkennung erfolgte jedoch dessen konsequente Umsetzung ${ }^{56}$ und mit einer solchen ist gerade aufgrund des weiten Regelungsumfangs der geplanten Richtlinie EEA erneut nicht zu rechnen. Hierdurch würden die mit der Richtlinie EEA eröffneten Möglichkeiten zur Beschleunigung und Vereinfachung des innerstaatlichen Verfahrens ungenutzt bleiben.

Hinsichtlich der Bearbeitung rechtshilferechtlicher Vorgänge im engeren Sinn bietet der Richtlinienentwurf einige Ansatzpunkte, die zu einer Beschleunigung und Vereinfachung des Verfahrens beitragen können, u.a. durch die Nutzung von Formblättern. Die Wirksamkeit wird jedoch abhängig sein von der Prüfung bestehender Geschäfts-

56 Gleß, in: Schomburg/Lagodny/Gleß/Hackner (Hrsg.), siehe Fn. 38, HT III, Rn. 57. 
prozesse der zuständigen Justizbehörden und deren Anpassung auf die Erfordernisse und Möglichkeiten der Richtlinie EEA hin. Die Gründe für die Versagung einer Anordnung sind im Vergleich zur traditionellen Rechtshilfe zwar reduziert, jedoch hat gerade der Umfang an Versagungsgründen im Richtlinienentwurf EEA im Vergleich zum Ursprungsentwurf nicht unerheblich zugenommen. Darüber hinaus ist nicht sicher, dass die formale Einführung von Fristen wirksam im Sinne einer Verfahrensbeschleunigung sein wird, da es an geeigneten Durchsetzungsmechanismen fehlt. Schließlich entstehen neue oder höhere verwaltungsmäßige Aufwände in Folge umfangreicher Bestätigungs-, Konsultations- und Unterrichtungspflichten. Dies schmälert im Weiteren die Chance auf mögliche Effizienzgewinne.

In einer vorläufigen Gesamtbetrachtung mag die mit der Richtlinie EEA beabsichtigte Abkehr von der traditionellen Rechtshilfe mit Ersuchen hin zu einem System der gegenseitigen Anerkennung mit Anordnungen zwar formal ein Paradigmenwechsel sein - in der Wirklichkeit deutscher Anwendungspraxis wird sich dies voraussichtlich aber als weit weniger erheblich darstellen. ${ }^{57}$

Dies mag erfreulich im Sinne der Aufrechterhaltung des bisherigen hohen rechtsstaatlichen Niveaus im deutschen Strafprozess - auch in grenzüberschreitenden Fällen - sein.

Gleichzeitig werden damit aber (zumindest auf deutscher Seite) die mit der Richtlinie verfolgten Ziele der Beschleunigung und Vereinfachung des transnationalen Strafverfahrens kaum oder gar nicht erreicht werden können. Wenn trotz entsprechender Regelungen in der geplanten Richtlinie EEA schon bei diesen Teilzielen eine Negativprognose abgegeben werden muss, wäre eine möglicherweise Beschränkung gegenwärtiger Gewährleistungen (bspw. in den Verfahrensrechten) mit der Einführung der Richtlinie EEA erst recht nicht hinnehmbar. ${ }^{58}$ Am Ende wird es jedoch auch am deutschen Gesetzgeber sein, in welches Verhältnis er die Teilziele der Richtlinie EEA mit seiner Umsetzung in innerstaatliches Recht bringt. Dabei liegt es gleichfalls in seinem Verantwortungsbereich, dem Anspruch aller Betroffenen im Strafverfahren gerecht zu werden, möglichst zügig die Unschuldsvermutung zu bestätigen oder zu widerlegen, um den Strafverfolgungsanspruch des Staates durchzusetzen und damit den Rechtsfrieden wiederherzustellen und zu bewahren.

57 So auch die Mehrzahl der von Wahl zu bisherigen Rechtsinstrumenten zur Verwirklichung des Grundsatzes der gegenseitigen Anerkennung interviewten Praktiker, Wahl (Fn. 51), 115 (121); Schierholt, ZIS 2010, 567 (567 f.).

58 Vgl. Rackow (Fn. 41), 117 (135): „Zwangsläufig führt nun der Versuch einer Steigerung der Effizienz der Strafverfolgung zu einer Gefährdung der strafprozessualen Waffengleichheit. Natürlich könnte man zu Gunsten des Richtlinienvorschlags [...] argumentieren, dass die Vereinfachung und somit Verbesserung der gegenseitigen Rechtshilfe in Strafsachen in der EU ja gerade das Anliegen ist.". 